\title{
PERTURBATION OF DOMAINS IN THE POMPEIU PROBLEM
}

\author{
TOSHIYUKI KOBAYASHI
}

ABSTRACT. An old problem in integral geometry called the Pompeiu problem is closely related to the existence of a solution of the overdetermined Neumann problem:

$$
(N)_{\lambda} \quad \begin{cases}\Delta u+\lambda u=0 & \text { in } \Omega, \\ \frac{\partial u}{\partial \nu}=0, u \equiv \text { constant } & \text { on } \partial \Omega .\end{cases}
$$

It is easy to see $(N)_{\lambda}$ holds if $\Omega$ is a ball. In this paper we shall give a quantitative estimate of the following statement in terms of one parameter family of domains and some special values of Bessel functions: If $\Omega$ is sufficiently 'close to' a ball and if $(N)_{\lambda}$ holds for a bounded $\lambda$, then $\Omega$ must be a ball.

\section{Introduction ANd Statements}

The study of the relations between the geometry of a given domain and the spectrum of the Laplace operator is very old and has been an area of active research. One of these problems is a free boundary problem called Schiffer's conjecture ([20], Problem 80), related to the Pompeiu problem which has originated in harmonic analysis ([15], [16]).

Suppose $\Omega$ is a bounded domain in $\mathbb{R}^{n}$ whose boundary $\partial \Omega$ is $C^{1}$ diffeomorphic to $S^{n-1}$. We associate the following three objects to $\Omega$ :

i) The null variety $\mathcal{N}(\Omega):=\left\{\zeta \in \mathbb{C}^{n}: \mathcal{F} \chi_{\Omega}(\zeta)=0\right\}$. Here $\mathcal{F} \chi_{\Omega}(\zeta)=$ $\int_{\Omega} e^{\sqrt{-1}\left(x_{1} \zeta_{1}+\ldots+x_{n} \zeta_{n}\right)} d x_{1} \ldots d x_{n}$ is the Fourier transform of the characteristic function $\chi_{\Omega}$, which is a holomorphic function of the variables $\zeta=\left(\zeta_{1}, \ldots, \zeta_{n}\right) \in \mathbb{C}^{n}$.

Supported by the NSF grant DMS-9100383. 
ii) An integral transform $T_{\Omega}: C\left(\mathbb{R}^{n}\right) \longrightarrow C(M(n))$ defined by $\left(T_{\Omega} f\right)(g)=$ $\int_{\Omega} f(g x) d x$. Here $M(n)=O(n) \ltimes \mathbb{R}^{n}$ is the Euclidean motion group.

iii) An overdetermined problem:

$$
(N)_{\lambda} \quad \begin{cases}\Delta u+\lambda u=0 & \text { in } \Omega, \\ \frac{\partial u}{\partial \nu}=0, u \equiv \text { constant } & \text { on } \partial \Omega .\end{cases}
$$

Here $\frac{\partial}{\partial \nu}$ stands for the outward normal vector field on $\partial \Omega$.

Then it is a well known result using an argument of spectral synthesis that these three objects are related with one another:

Fact 1.1. ([7], [18]) In the above setting, the following three conditions on $\Omega$ are equivalent:

(1) There exists $r>0$ such that $\mathcal{N}(\Omega) \supset S_{\mathbb{C}}(0: r)$.

(2) $\operatorname{Ker} T_{\Omega} \neq\{0\}$.

(3) There exists $\lambda>0$ and a nontrivial solution $u$ of $(N)_{\lambda}$.

Here, $S_{\mathbb{C}}(a: r):=\left\{\zeta \in \mathbb{C}^{n}: \sum_{j=1}^{n}\left(\zeta_{j}-a_{j}\right)^{2}=r^{2}\right\}$ for $a=\left(a_{1}, \ldots, a_{n}\right) \in \mathbb{R}^{n}$ and $r \in \mathbb{R}$. (1) and (3) are related by $\lambda=r^{2}$.

A ball in $\mathbb{R}^{n}$ satisfies the above three conditions. In fact, if $\Omega$ is the unit ball, then

$$
\mathcal{F} \chi_{\Omega}(\zeta)=f_{\frac{n}{2}}\left(\sqrt{{\zeta_{1}^{2}}^{2}+\ldots+\zeta_{n}^{2}}\right), \quad\left(\zeta=\left(\zeta_{1}, \ldots, \zeta_{n}\right) \in \mathbb{C}^{n}\right)
$$

where $f_{\nu}(z):=(2 \pi)^{\frac{n}{2}} \frac{J_{\nu}(z)}{z^{\nu}}$ is a holomorphic function of $z \in \mathbb{C}$ (Lemma 2.2 (1)). Hence,

$$
\begin{aligned}
\mathcal{N}(\Omega) & \supset S_{\mathbb{C}}(0: r), \\
\operatorname{Ker} T_{\Omega} & \ni f_{\frac{n}{2}-1}\left(r \sqrt{x_{1}^{2}+\ldots+x_{n}^{2}}\right),
\end{aligned}
$$

$$
f_{\frac{n}{2}-1}\left(r \sqrt{x_{1}^{2}+\ldots+x_{n}^{2}}\right) \text { is a solution to }(N)_{r^{2}},
$$

whenever $r$ is a positive zero of the Bessel function $J_{\frac{n}{2}}(z)$ (there exist countably many).

Conversely, it has been a long standing conjecture (the Pompeiu problem, Schiffer's conjecture) that a ball would be the only domain satisfying one of 
(any of) (1)-(3) in Fact 1.1. Berenstein [3], Aviles [2] (see also [4] Proposition 1 ), and Brown and Kahane [6] have made progress regarding $(N)_{\lambda}$ which might be summarized as follows: For simplicity, suppose $\Omega$ is convex in $\mathbb{R}^{2}$ (this assumption can be weakened in (1.4.1) and (1.4.2)).

(1.4.1) If $(N)_{\lambda}$ holds for infinitely many $\lambda_{j}$, then $\Omega$ is a ball.

(1.4.2) If $(N)_{\lambda}$ holds and $\lambda \leq \nu_{7}$, the seventh Neumann eigenvalue, then also $\Omega$ is a ball.

(1.4.3) If $(N)_{\lambda}$ holds and $n=2$, then $2 \min _{\theta \in S^{1}} H_{\Omega}(\theta)>\max _{\theta \in S^{1}} H_{\Omega}(\theta)$, where the breadth function $H_{\Omega}(\theta):=\sup _{x \in \Omega}(x, \theta)-\inf _{x \in \Omega}(x, \theta)$.

For further progress and some survey related to this conjecture, see [5], [9], and [14] and the references therein.

Loosely speaking, the result (1.4.3) of Brown and Kahane asserts that a long thin convex domain in $\mathbb{R}^{2}$ ('far from' being a ball) never satisfies the conditions (1) - (3) in Fact 1.1. In contrast to this, our concern in this paper will be with the case where $\Omega$ is sufficiently 'close to' a ball. In order to define the 'closeness' and to give a quantitative estimate on how perturbations of a ball affect the properties in Fact 1.1, we need to take parallel translations and similarity transforms into account because they do not affect the properties in Fact 1.1. So, we first define unessential perturbations as follows.

Given a continuous function $g: S^{n-1} \longrightarrow \mathbb{R}_{+}=\{x \in \mathbb{R}: x>0\}$, we define a star-shaped region $\Omega(g)$ with respect to the origin by

$$
\Omega(g):=\left\{\rho \cdot \eta \in \mathbb{R}^{n}: \eta \in S^{n-1}, 0 \leq \rho<g(\eta)\right\}
$$

Suppose $g:[0, T] \times S^{n-1} \longrightarrow \mathbb{R}_{+}$is a $C^{1}$-map such that $g(0, \eta) \equiv 1\left(\eta \in S^{n-1}\right)$. Then $\Omega_{t}:=\Omega(g(t, \cdot))$ forms a family of domains parametrized by $t \in[0, T]$ with $\Omega_{0}=B(0: 1)$, the unit ball.

We call $\left\{\Omega_{t}\right\}$ unessential if there exist $a \in \mathbb{R}$ and $b \in \mathbb{R}^{n}$ such that $g_{t}(0, \eta)=$ $a+(b, \eta)\left(g_{t}:=\frac{\partial g}{\partial t}\right)$ (i.e. $g_{t} \in E_{0}+E_{1}$ with the notation of Lemma 2.5 on spherical harmonics). This means that $\left\{\Omega_{t}\right\}$ is degenerate at $t=0$ up to similarity transformations and parallel translations (see $\S 5$ ).

Fix $0<T, 0<\alpha \leq 1$ and $0 \ll R$. For a $C^{1, \alpha}$ function $g:[0, T] \times S^{n-1} \longrightarrow$ 
$\mathbb{R}_{+}$we shall define (see (2.6.4), (3.3.3)):

$$
[g]_{R, \alpha}:=\frac{\left|g_{t}(0, \cdot)\right|_{R}}{\|g\|_{1+\alpha}}(\geq 0)
$$

by using spherical harmonics and Bessel functions with the following property (see (2.6.5)):

$$
[g]_{R, \alpha}=0 \Leftrightarrow\{\Omega(g(t, \cdot))\} \text { is unessential. }
$$

We put $B(a: r)=\left\{x \in \mathbb{R}^{n}: \sum_{j=1}^{n}\left(x_{j}-a_{j}\right)^{2}<r^{2}\right\}, S(a: r)=\left\{x \in \mathbb{R}^{n}:\right.$ $\left.\sum_{j=1}^{n}\left(x_{j}-a_{j}\right)^{2}=r^{2}\right\}$ for $a=\left(a_{1}, \ldots, a_{n}\right) \in \mathbb{R}^{n}, r>0 . \mathcal{N}(\Omega)_{\mathbb{R}}=\mathcal{N}(\Omega) \cap \mathbb{R}^{n}$ Now we are ready to state our main result:

Theorem. Let $R \gg 0$. There exists a constant $C(n, R)>0$ with the following property: Suppose $0<T, 0<\alpha \leq 1$ and that $\Omega_{t} \equiv \Omega(g(t, \cdot)) \quad(0 \leq t \leq T)$ is a family of domains in $\mathbb{R}^{n}$ given by a $C^{1, \alpha}$ map $g:[0, T] \times S^{n-1} \longrightarrow \mathbb{R}_{+}$satisfying $g(0, \cdot) \equiv 1$ and $\left|g_{t}(0, \eta)\right| \leq 1\left(\eta \in S^{n-1}\right)$. If there exist $t_{0} \in \mathbb{R}, x \in \mathbb{R}^{n}$ and $r>0$ such that

$$
\begin{gathered}
0 \leq t_{0}<\min \left(T,\left(C(n, R)[g]_{R, \alpha}\right)^{\frac{1}{\alpha}}\right), \\
\mathcal{N}\left(\Omega_{t_{0}}\right)_{\mathbb{R}} \cap B(0: R) \supset S(x: r),
\end{gathered}
$$

then $t_{0}=0$ and so $\Omega_{t_{0}}$ is a ball.

Remark. Actually it suffices to assume $R \geq j\left(\frac{n}{2}, 1\right)$ the first positive zero of $J_{\frac{n}{2}}(z)$, when the constant $C(n, R)$ is given in (4.5.2). In Proposition 4.4 we drop the technical assumption $\left|g_{t}(0, \eta)\right| \leq 1 \quad\left(\eta \in S^{n-1}\right)$. A small price to pay is the condition (4.4.1b) on $t_{0}$ is somewhat complicated (see also Lemma 4.5).

Corollary 1. Let $R \gg 0$ and retain the setting as in the theorem above. Assume that there exist $\lambda_{0}, t_{0} \in \mathbb{R}$ and $u \in C^{2}\left(\Omega_{t_{0}}\right) \cap C^{1}\left(\overline{\Omega_{t_{0}}}\right)$ such that

$$
\begin{aligned}
& 0<\lambda_{0}<R^{2}, \\
& 0 \leq t_{0}<\min \left(T,\left(C(n, R)[g]_{R, \alpha}\right)^{\frac{1}{\alpha}}\right), \\
& u \neq 0 \text { is a solution of }(N)_{\lambda_{0}} .
\end{aligned}
$$

Then $t_{0}=0$ and $\Omega_{t_{0}}$ is the unit ball. 
In a slightly different formulation we may define a perturbation of domains $\left\{\Omega_{t}\right\}$ by giving the boundaries by means of a continuous map $G:[0, T] \times$ $S^{n-1} \longrightarrow \mathbb{R}^{n}$ with $G(0, \eta)=\eta\left(\eta \in S^{n-1}\right)$ such that

$$
\partial \Omega_{t}=G\left(t, S^{n-1}\right):=\text { Image }\left(G(t, \cdot): S^{n-1} \longrightarrow \mathbb{R}^{n}\right) .
$$

If $G$ is in the $C^{1}$ class, then we find a uniquely defined function $g_{G}:\left[0, T^{\prime}\right] \times$ $S^{n-1} \longrightarrow \mathbb{R}_{+}\left(0<T^{\prime} \leq T\right)$ such that $\Omega_{t}=\Omega\left(g_{G}(t, \cdot)\right)$ (see Lemma 5.4). In $\S 5$ we discuss a geometric aspect of unessential perturbations. In particular, $\left\{\Omega_{t}\right\}$ is unessential if and only if there exist $0<T^{\prime \prime} \leq T$ and $C^{1}$-maps

$$
\left\{\begin{array}{ll}
a:\left[0, T^{\prime \prime}\right] \rightarrow \mathbb{R}, & a(0)=1, \\
b:\left[0, T^{\prime \prime}\right] \rightarrow \mathbb{R}^{n}, & b(0)=t^{t}(0, \ldots, 0), \\
\varphi:\left[0, T^{\prime \prime}\right] \times S^{n-1} \rightarrow S^{n-1}, & \varphi(0, \omega) \equiv \omega, \\
& \varphi(t, \cdot) \text { is a } C^{1} \text {-diffeomorphism of } \\
& S^{n-1}
\end{array},\right.
$$

such that $\widetilde{G}(t, \omega):=a(t) G(t, \varphi(t, \omega))+b(t)$ is degenerate at $t=0$ in the sense that $\left.\frac{\partial}{\partial t}\right|_{t=0} \widetilde{G}(t, \omega) \equiv 0$ (Proposition 5.7). Because of the ambiguity of the map $G$ up to $\operatorname{Map}\left([0, T], \operatorname{Homeo}\left(S^{n-1}\right)\right)$, we present only a weaker version of Corollary 1 by means of a sequence $t_{j}$ in this formulation, instead of giving an explicit estimate of a single $t_{0}$ as in Corollary 1.

Corollary 2. Let $0<T$ and $0<\alpha \leq 1$. Suppose $\left\{\Omega_{t}\right\}(0 \leq t \leq T)$ is not an unessential family of domains in $\mathbb{R}^{n}$ given by a $C^{1, \alpha}$ map $G:[0, T] \times S^{n-1} \longrightarrow$ $\mathbb{R}^{n}$ with $G(0, \eta) \equiv \eta \quad\left(\eta \in S^{n-1}\right)$. Assume that there exists a sequence $t_{j} \in$ $[0, T], \lambda_{j} \in \mathbb{R} \quad(j \in \mathbb{N})$ and $u_{j} \in C^{2}\left(\Omega_{t_{j}}\right) \cap C^{1}\left(\overline{\Omega_{t_{j}}}\right)$ such that

$$
\left\{\begin{array}{l}
\lim _{j \rightarrow \infty} t_{j}=0, \\
\sup _{j \in \mathbb{N}}\left|\lambda_{j}\right|<\infty \\
u_{j} \text { is a solution of }(N)_{\lambda_{j}} \text { in } \Omega_{t_{j}} .
\end{array}\right.
$$

Then there exists $n_{0} \in \mathbb{N}$ such that $\Omega_{t_{j}}$ is a ball for any $j \geq n_{0}$.

We finally mention that the results here were announced in [11] Theorem 3 in 1986 (see also [12] Chapter 3 and [14] Theorem 5.12) with the $C^{2}$ assumption on the parameter. Recently C. Berenstein brought to our attention a preprint of Agranovsky [1]. There Agranovsky gives a similar result to Corollary 2 assuming that the dimension $n=2$ and assuming the existence of a solution 
to $(N)_{\lambda_{t}}$ for all $t$ with the condition that both the boundary $\partial \Omega_{t}$ and the eigenvalues $\lambda_{t}$ depend analytically on the parameter $t$, though his result covers unessential perturbations (cf. Remark (5.10)). His approach using Riemann's mapping theorem for $\mathbb{C} \simeq \mathbb{R}^{2}$ is quite different from ours.

\section{PRELiminary RESUlts ON}

\section{BESSEL FUNCTIONS AND SPHERICAL HARMONICS}

In this section we give a short survey on Bessel functions and spherical harmonics on $S^{n-1}$ that will be used in later sections.

For $\nu \in \mathbb{C}$ and $z \in \mathbb{C} \backslash(-\infty, 0]$, the $\nu$-th Bessel function is defined by

$$
\begin{aligned}
J_{\nu}(z): & =\left(\frac{z}{2}\right)^{\nu} \sum_{n=0}^{\infty} \frac{(-1)^{n}\left(\frac{z}{2}\right)^{2 n}}{n ! \Gamma(\nu+n+1)} \\
& =\frac{\left(\frac{z}{2}\right)^{\nu}}{\sqrt{\pi} \Gamma\left(\nu+\frac{1}{2}\right)} \int_{-1}^{1} e^{\sqrt{-1} z t}\left(1-t^{2}\right)^{\nu-\frac{1}{2}} d t .
\end{aligned}
$$

If we put $\theta=z \frac{d}{d z}$, then both $J_{\nu}(z)$ and $J_{-\nu}(z)$ are the solutions of the ordinary differential equation $\left(\theta^{2}+\left(z^{2}-\nu^{2}\right)\right) f(z)=0$.

Here is a collection of some basic properties of the Bessel function:

\section{Lemma 2.2.}

1) $\frac{J_{\nu}(z)}{z^{\nu}}$ is an entire function of $z \in \mathbb{C}$ with $\left.\frac{J_{\nu}(z)}{z^{\nu}}\right|_{z=0}=\frac{1}{2^{\nu} \Gamma(\nu+1)}$.

2) (see [8], (7.5.38), (7.15.42)) Let $z \in \mathbb{C}$. We have

$$
\begin{aligned}
& \sum_{n=-\infty}^{\infty} J_{n}(z)^{2}=1 . \\
& \sum_{n=0}^{\infty}(2 n+1) J_{n+\frac{1}{2}}(z)^{2}=\frac{2 z}{\pi} .
\end{aligned}
$$

3) For real $\nu>-1, J_{\nu}(z)$ has only real zeros (Lommel, see [17], pp.482-483). Let denote by $j(\nu, 1)$ the first positive zero of $J_{\nu}(z)(\nu>0)$. Then $j(\nu, 1)>\nu$ ([17], p.485).

4) (Siegel, see [17], p.484) $J_{\nu}(z)$ and $J_{\nu+m}(z)\left(m \in \mathbb{N}_{+}\right)$have no common zeros other than zero (Bourget's conjecture). 
The first and the last statements in Lemma 2.2 imply the following lemma, in which the constants $\varepsilon(n, R)$ and $\delta(n, R)$ will be used in a quantitative estimate of our Theorem.

Lemma 2.3. Let $j\left(\frac{n}{2}, k\right) \quad\left(k \in \mathbb{N}_{+}\right)$be the $k$-th positive zero of the Bessel function $J_{\frac{n}{2}}(x)$. We fix $R \geq j\left(\frac{n}{2}, 1\right)$ and define $k_{R} \in \mathbb{N}_{+}$by

$$
0=j\left(\frac{n}{2}, 0\right)<j\left(\frac{n}{2}, 1\right)<\ldots<j\left(\frac{n}{2}, k_{R}\right) \leq R<j\left(\frac{n}{2}, k_{R}+1\right) .
$$

We define an entire function of $z \in \mathbb{C}$ by

$$
f(z):=(2 \pi)^{\frac{n}{2}} \frac{J_{\frac{n}{2}}(z)}{z^{\frac{n}{2}}} .
$$

Put

$$
W(R):=\{z \in \mathbb{C}: 0 \leq \operatorname{Re} z \leq R,|\operatorname{Im} z| \leq 1\} .
$$

Then there exist $\varepsilon \equiv \varepsilon(n, R)>0$ and $\delta \equiv \delta(n, R)>0$ such that the following three conditions hold.

(2.3.2) $\delta(n, R)<\frac{1}{2} \min _{1 \leq k \leq n}\left(j\left(\frac{n}{2}, k\right)-j\left(\frac{n}{2}, k-1\right)\right)$.

(2.3.3) $\zeta \in W(R) \cap B\left(j\left(\frac{n}{2}, k\right): \delta\right)$ for some $k \in\left\{1, \ldots, k_{R}\right\}$, then

$$
|f(z)| \geq \varepsilon(n, R)\left|z-j\left(\frac{n}{2}, k\right)\right| .
$$

(2.3.4) If $z \in W(R) \backslash \bigcup_{k=1}^{k_{R}} B\left(j\left(\frac{n}{2}, k\right): \delta\right)$, then

$$
|f(z)| \geq \varepsilon(n, R) \delta(n, R) .
$$

Remark 2.4. We might take $\delta(n, R)=1$, however, we do not pursue this kind of numerical estimates of the Bessel function in this paper. On the other hand, we can and do assume

$$
\begin{aligned}
& \varepsilon(n, R) \leq \delta(n, R) \leq 1, \\
& \varepsilon(n, R) \leq \omega_{n-1} .
\end{aligned}
$$

Here we define

$$
\omega_{n-1}:=\operatorname{Vol}\left(S^{n-1}\right)=\frac{2 \pi^{\frac{n}{2}}}{\Gamma\left(\frac{n}{2}\right)}
$$

Next, we review spherical harmonics. We induce a Riemannian metric $g$ on $S^{n-1}$ from the standard metric on $\mathbb{R}^{n}$. Denote by $\Delta_{S^{n-1}}$ the Laplace-Beltrami 
operator on $S^{n-1}$ with respect to $g$. Then the Laplace operator $\Delta \equiv \Delta_{\mathbb{R}^{n}}$ on $\mathbb{R}^{n}$ is represented as $\Delta=\frac{\partial^{2}}{\partial r^{2}}+\frac{n-1}{r} \frac{\partial}{\partial r}+\frac{1}{r^{2}} \Delta_{S^{n-1}}$ in the spherical coordinate $x=r \omega\left(r>0, \omega \in S^{n-1}\right)$. We write $L^{2}\left(S^{n-1}\right)$ for the Hilbert space of square integrable functions with respect to the measure given by the metric $g$. For each non-negative integer $k \in \mathbb{N}$ we define an eigenspace

$$
E_{k}:=\left\{f \in C^{\infty}\left(S^{n-1}\right): \Delta_{S^{n-1}} f=-k(k+n-2) f\right\} \subset L^{2}\left(S^{n-1}\right) .
$$

The orthogonal group $O(n)$ naturally acts on the unit sphere $S^{n-1}$. This action is transitive and we have $O(n) / O(n-1) \simeq S^{n-1}$. Since this action is isometric, $\Delta_{S^{n-1}}$ belongs to the algebra of $O(n)$-invariant differential operators and so $E_{k}$ is an $O(n)$-submodule of $L^{2}\left(S^{n-1}\right)$, on which $O(n)$ acts unitarily by $f(x) \mapsto f\left(g^{-1} x\right)$ for $f \in L^{2}\left(S^{n-1}\right), x \in S^{n-1}$ and $g \in O(n)$.

In fact, it turns out that each $E_{k}$ is an irreducible $O(n)$-module and the eigenvalue of $\Delta_{S^{n-1}}$ is given as an evaluation of the Casimir operator. Here are classical results on spherical harmonics:

Lemma 2.5. (see [10], Introduction, Theorem 3.1 and Lemma 3.6)

1) $L^{2}\left(S^{n-1}\right)=\sum_{k=0}^{\infty} \oplus E_{k}$, (orthogonal Hilbert space decomposition).

2) $E_{k}$ is irreducible as an $O(n)$-module.

3) $E_{k}$ is the $\mathbb{C}$-span of

$$
\left\{\left(a_{1} x_{1}+\cdots+a_{n} x_{n}\right)^{k}: a_{j} \in \mathbb{C}, \sum_{j=1}^{n}{a_{j}}^{2}=0,\left(x_{1}, \ldots, x_{n}\right) \in S^{n-1}\right\} .
$$

In particular,

$$
\begin{aligned}
& E_{0}=\mathbb{C} \cdot 1, \\
& E_{1}=\mathbb{C} x_{1} \oplus \ldots \oplus \mathbb{C} x_{n} .
\end{aligned}
$$

4) (see also [8], p. 247, (11.4.22)) For $h \in L^{2}\left(S^{n-1}\right), r \in \mathbb{C}$ and $\omega \in S^{n-1}$ we put

$$
\hat{h}(r, \omega):=\int_{S^{n-1}} e^{\sqrt{-1} r(\eta, \omega)} h(\eta) d \eta .
$$

If $h \in E_{k}(k \in \mathbb{N})$, then we have for $\omega \in S^{n-1}$ and $r \in \mathbb{C}$

$$
\hat{h}(r, \omega)=(2 \pi)^{\frac{n}{2}} \sqrt{-1}^{k} h(\omega) \frac{J_{k+\frac{n}{2}-1}(r)}{r^{\frac{n}{2}-1}} .
$$


We introduce a family of seminorms $|\cdot|_{r}^{\prime}$ on $L^{2}\left(S^{n-1}\right)$ parametrized by $r>0$ by

$$
|h|_{r}^{\prime}:=\left\{\sum_{k=1}^{\infty}\left\|h_{k}\right\|_{L^{2}\left(S^{n-1}\right)}^{2} J_{k+\frac{n}{2}-1}(r)^{2}\right\}^{\frac{1}{2}}
$$

for $h=\sum_{k=0}^{\infty} h_{k} \in L^{2}\left(S^{n-1}\right)=\sum_{k=0}^{\infty} \oplus E_{k}$. If $h \in \sum_{k=1}^{\infty} \oplus E_{k}$ (i.e. $h_{0}=0$ ), then it follows from (2.5.4) that

$$
\|\hat{h}(r, \omega)\|_{L^{2}\left(S^{n-1}\right)}=(2 \pi)^{\frac{n}{2}} r^{1-\frac{n}{2}}|h|_{r}^{\prime}
$$

Since $\sum_{k=0}^{\infty} J_{k+\frac{n}{2}-1}(r)^{2}<\max (1, r)$ (see Lemma $\left.2.2(2)\right)$ if $\mathbb{N} \ni n \geq 2$, we have

$$
|h|_{r}^{\prime} \leq\|h\|_{L^{2}\left(S^{n-1}\right)} \max \left(1, r^{\frac{1}{2}}\right) .
$$

Let $j(\nu, k)\left(k \in \mathbb{N}_{+}\right)$be the positive zeros of $J_{\nu}(z)$ arranged in ascending order. For $R \geq j\left(\frac{n}{2}, 1\right)$ let $k_{R} \in \mathbb{N}_{+}$be the integer such that

$$
0<j\left(\frac{n}{2}, 1\right)<j\left(\frac{n}{2}, 2\right)<\ldots<j\left(\frac{n}{2}, k_{R}\right) \leq R<j\left(\frac{n}{2}, k_{R}+1\right) .
$$

For $h \in L^{2}\left(S^{n-1}\right)$, we define

$$
|h|_{R}:=\min _{1 \leq k \leq k_{R}}|h|_{j\left(\frac{n}{2}, k\right)}^{\prime} .
$$

It follows from Lemma $2.2(4)$ that

$$
|h|_{R}=0 \quad \Leftrightarrow h \in E_{0}+E_{1} .
$$

\section{SOME ESTIMATES OF ERROR TERMS.}

In this section we give the first and second terms of the expansion of $\mathcal{F} \chi_{\Omega_{t}}(\zeta$. $\omega)$, the Fourier transform of a family of domains $\left\{\Omega_{t}\right\}$ in $\mathbb{R}^{n}$, and also give an estimate of the error term.

We define a function $F: \mathbb{C} \times \mathbb{C} \rightarrow \mathbb{C}$ by

$$
F(s, u):=\frac{(-1)^{n}(n-1) !}{(\sqrt{-1} u)^{n-1}}\left\{1-e^{\sqrt{-1} s u} \sum_{k=0}^{n-1} \frac{(-\sqrt{-1} s u)^{k}}{k !}\right\} .
$$

By a simple calculation we have

$$
\frac{\partial F}{\partial s}(s, u)=s^{n-1} e^{\sqrt{-1} s u},
$$


and thus

$$
\int_{0}^{s} e^{\sqrt{-1} \rho u} \rho^{n-1} d \rho=F(s, u) .
$$

By means of this function $F$ we have a formula of $\mathcal{F} \chi_{\Omega(g)}(\zeta)$ :

Lemma 3.2. Suppose $g: S^{n-1} \rightarrow \mathbb{R}_{+}$is a continuous function and that $\Omega(g)$ is a star-shaped region with respect to the origin as in (1.5). Then we have a formula

$$
\mathcal{F}_{\chi \Omega(g)}(\zeta)=\int_{S^{n-1}} F(g(\eta),(\eta, \zeta)) d \eta, \quad \zeta \in \mathbb{C}^{n}
$$

Proof. From definition we have

$$
\begin{aligned}
\mathcal{F}_{\Omega(g)}(\zeta) & =\int_{\Omega(g)} e^{\sqrt{-1}(x, \zeta)} d x \\
& =\int_{S^{n-1}} \int_{0}^{g(\eta)} e^{\sqrt{-1} r(\eta, \zeta)} r^{n-1} d r d \eta \\
& =\int_{S^{n-1}} F(g(\eta),(\eta, \zeta)) d \eta . \quad \square
\end{aligned}
$$

We fix $0<T$ and $0<\alpha \leq 1$. For a $C^{0, \alpha}$-map $f:[0, T] \rightarrow \mathbb{R}$, we put

$$
\|f\|_{C^{0, \alpha}([0, T])}:=\sup _{0 \leq t \leq T}|f(t)|+\sup _{0 \leq s<t \leq T} \frac{|f(t)-f(s)|}{|t-s|^{\alpha}}(<\infty) .
$$

Next, suppose $g:[0, T] \times S^{n-1} \rightarrow \mathbb{R}$ is a $C^{1, \alpha}$-map. We put

$$
\begin{aligned}
& \|g\|_{\infty}:=\sup _{\substack{0 \leq t \leq T \\
\eta \in S^{n-1}}}|g(t, \eta)|, \\
& \|g\|_{0+\alpha}:=\sup _{\eta \in S^{n-1}}\|g(\cdot, \eta)\|_{C^{0, \alpha}([0, T])}, \\
& \|g\|_{1+\alpha}:=\|g\|_{\infty}+\left\|g_{t}\right\|_{0+\alpha},
\end{aligned}
$$

where $g_{t}(t, \eta):=\frac{\partial}{\partial t} g(t, \eta)$.

Lemma 3.4. Let $0<T$ and $0<\alpha \leq 1$. Suppose $g:[0, T] \times S^{n-1} \rightarrow \mathbb{R}_{+}$is a $C^{1, \alpha}$ map such that

$$
g(0, \eta) \equiv 1 \quad \text { for } \eta \in S^{n-1}
$$


We write $\Omega_{t}=\Omega(g(t, \cdot))$ (see (1.5)). In particular, $\Omega_{0}$ is the unit ball. We put

$$
Y(g: r, T, \alpha):=\frac{1}{1+\alpha} \sup _{\substack{\eta \in S^{n-1} \\ \omega \in S^{n-1}}}\left\|g(t, \eta)^{n-1} e^{\sqrt{-1} r g(t, \eta)(\eta, \omega)} g_{t}(t, \eta)\right\|_{C^{0, \alpha}([0, T])} .
$$

If $\zeta \in \mathbb{C}, r \geq 0$ and $\omega \in S^{n-1}$, then we have (see (2.4.3)for the definition of $\left.\omega_{n-1}\right)$

$$
\begin{aligned}
& \left|\mathcal{F} \chi_{\Omega_{t}}(\zeta \cdot \omega)-\mathcal{F} \chi_{\Omega_{0}}(\zeta \cdot \omega)\right| \leq t\|g\|_{\infty}^{n-1}\left\|g_{t}\right\|_{\infty} \omega_{n-1} e^{|\operatorname{Im} \zeta|\|g\|_{\infty}} \\
& \left|\mathcal{F} \chi_{\Omega_{t}}(r \cdot \omega)-\mathcal{F} \chi_{\Omega_{0}}(r \cdot \omega)-t \frac{\partial}{\partial t}\right|_{t=0} \mathcal{F} \chi_{\Omega_{t}}(r \cdot \omega) \mid \\
& \leq t^{1+\alpha} \omega_{n-1} Y(g: r, T, \alpha) \text {. }
\end{aligned}
$$

Proof. Let $\zeta \in \mathbb{C}$ and $\omega \in S^{n-1}$. By Lemma 3.2 we have

$$
\mathcal{F} \chi_{\Omega_{t}}(\zeta \cdot \omega)=\int_{S^{n-1}} F(g(t, \eta), \zeta(\eta, \omega)) d \eta
$$

Then, from (3.1.2) we have

$$
\frac{\partial}{\partial t} \mathcal{F} \chi_{\Omega_{t}}(\zeta \cdot \omega)=\int_{S^{n-1}} g(t, \eta)^{n-1} g_{t}(t, \eta) e^{\sqrt{-1} g(t, \eta) \zeta(\eta, \omega)} d \eta
$$

Thus,

$$
\begin{aligned}
\left|\mathcal{F} \chi_{\Omega_{t}}(\zeta \cdot \omega)-\mathcal{F} \chi_{\Omega_{0}}(\zeta \cdot \omega)\right| & \leq t \sup _{0 \leq s \leq t}\left|\frac{\partial}{\partial s} \mathcal{F} \chi_{\Omega_{s}}(\zeta \cdot \omega)\right| \\
& \leq t \omega_{n-1}\|g\|_{\infty}^{n-1}\left\|g_{t}\right\|_{\infty} e^{|\operatorname{Im} \zeta|\|g\|_{\infty}}
\end{aligned}
$$

which shows the first inequality (3.4.4).

It follows from the definition of $Y(g: r, T, \alpha)$ and (3.4.5) that

$$
\left|\frac{\partial}{\partial t} \mathcal{F} \chi_{\Omega_{t}}(r \cdot \omega)-\frac{\partial}{\partial t}\right|_{t=0} \mathcal{F} \chi_{\Omega_{t}}(r \cdot \omega) \mid \leq(1+\alpha) Y(g: r, T, \alpha) \omega_{n-1} t^{\alpha}
$$

for $0 \leq t \leq T, \omega \in S^{n-1}$ and $r>0$. Therefore, we have

$$
\begin{aligned}
& \left|\mathcal{F} \chi_{\Omega_{t}}(r \cdot \omega)-\mathcal{F} \chi_{\Omega_{0}}(r \cdot \omega)-t \frac{\partial}{\partial s}\right|_{s=0} \mathcal{F} \chi_{\Omega_{s}}(r \cdot \omega) \mid \\
= & \left|\int_{0}^{t}\left\{\frac{\partial}{\partial s} \mathcal{F} \chi_{\Omega_{s}}(r \cdot \omega)-\left.\frac{\partial}{\partial s}\right|_{s=0} \mathcal{F} \chi_{\Omega_{s}}(r \cdot \omega)\right\} d s\right| \\
\leq & t^{1+\alpha} Y(g: r, T, \alpha) \omega_{n-1} \cdot \square
\end{aligned}
$$

We shall need the following estimate of $Y$ in $\S 4$. 
Lemma 3.5. Retain the setting of Lemma 3.4. We define $\tilde{g}:[0, T] \times S^{n-1} \times$ $\mathbb{R} \rightarrow \mathbb{R}$ by

$$
\tilde{g}(t, \eta, a):=(1+a t) g(t, \eta)
$$

Assume

$$
0<T \leq 1 \text { and }|a| T \leq 1
$$

Then

(3.5.3) $Y(\tilde{g}(\cdot, \cdot, a): r, T, \alpha) \leq 2^{n+1}(n+r)\|g\|_{\infty}^{n-1}\|g\|_{0+\alpha}\|g\|_{1+\alpha}(1+|a|)^{2}$.

Proof. First we treat the case $a=0$. Fix $r>0$ and $\eta, \omega \in S^{n-1}$. We put $h(x):=x^{n-1} e^{\sqrt{-1} x r(\eta, \omega)}$. Then

$$
\begin{aligned}
& \|h(g(\cdot, \eta))\|_{C^{0, \alpha}([0, T])} \\
\leq & \sup _{0 \leq t \leq T}|h(g(t, \eta))|+\sup _{0 \leq t \leq T}\left|h_{t}(g(t, \eta))\right| \sup _{0 \leq s<t \leq T} \frac{|g(t, \eta)-g(s, \eta)|}{|t-s|^{\alpha}} \\
\leq & \|g\|_{\infty}^{n-1}+\left\{(n-1)\|g\|_{\infty}^{n-2}+\|g\|_{\infty}^{n-1} r\right\}\|g\|_{0+\alpha} \\
\leq & (n+r)\|g\|_{\infty}^{n-1}\|g\|_{0+\alpha} .
\end{aligned}
$$

Here we have used $\|g\|_{\infty} \geq 1$ in the last inequality. Hence

$$
\begin{aligned}
Y(g: r, T, \alpha) & =\frac{1}{1+\alpha} \sup _{\eta, \omega \in S^{n-1}}\left\|h(g(\cdot, \eta)) g_{t}(\cdot, \eta)\right\|_{C^{0, \alpha}([0, T])} \\
& \leq\left\|g_{t}\right\|_{0+\alpha} \sup _{\eta, \omega \in S^{n-1}}\|h(g(\cdot, \eta))\|_{C^{0, \alpha}([0, T])} \\
& \leq(n+r)\|g\|_{\infty}^{n-1}\|g\|_{0+\alpha}\left\|g_{t}\right\|_{0+\alpha}
\end{aligned}
$$

Next, let us estimate $Y$ for $\tilde{g}(t, \eta, a)=(1+a t) g(t, \eta)$ in terms of $g$. We assume $T \leq 1$ and $|a| T \leq 1$. Since

$$
\begin{aligned}
\tilde{g}_{t}(t, \eta, a) & =a g(t, \eta)+(1+a t) g_{t}(t, \eta), \\
\|1+a t\|_{0+\alpha} & \leq\|1+a t\|_{0+1} \leq 2+|a|,
\end{aligned}
$$


we have

$$
\begin{aligned}
\|\tilde{g}\|_{\infty} & \leq 2\|g\|_{\infty} \\
\|\tilde{g}(\cdot, \cdot, a)\|_{0+\alpha} & \leq\|1+a t\|_{0+\alpha}\|g\|_{0+\alpha} \\
& \leq(2+|a|)\|g\|_{0+\alpha} \\
\left\|\tilde{g}_{t}(\cdot, \cdot, a)\right\|_{0+\alpha} & \leq|a|\|g\|_{0+\alpha}+\|1+a t\|_{0+\alpha}\left\|g_{t}\right\|_{0+\alpha} \\
& \leq 2(1+|a|)\|g\|_{1+\alpha} .
\end{aligned}
$$

Applying (3.5.4) to $\tilde{g}$, we have

$$
\begin{aligned}
Y(\tilde{g}(\cdot, \cdot, a): r, T, \alpha) & \leq(n+r) 2^{n-1}\|g\|_{\infty}^{n-1}(2+|a|)\|g\|_{0+\alpha} 2(1+|a|)\|g\|_{1+\alpha} \\
& \leq 2^{n+1}(n+r)(1+|a|)^{2}\|g\|_{\infty}^{n-1}\|g\|_{0+\alpha}\|g\|_{1+\alpha} . \quad \square
\end{aligned}
$$

\section{Proof of Theorem}

Now we are ready to prove our main Theorem. We start with the following:

Lemma 4.1. In the setting of Lemma 3.4, let $\rho$ be a positive zero of $J_{\frac{n}{2}}(x)$. Assume

$$
0 \leq t<\min \left(T,\left(\frac{(2 \pi)^{\frac{n}{2}}\left|g_{t}(0, \cdot)\right|_{\rho}^{\prime}}{\rho^{\frac{n}{2}-1} \omega_{n-1}{ }^{\frac{3}{2}} Y(g: \rho, T, \alpha)}\right)^{\frac{1}{\alpha}}\right),
$$

(see (2.6.1) and (3.4.2) for notation). Then $\mathcal{N}\left(\Omega_{t}\right) \supset S(0: \rho)$ if and only if $t=0$. Here we write $\Omega_{t}=\Omega(g(t, \cdot))$.

Proof. If $t=0$, then $\mathcal{F} \chi_{\Omega_{0}}(r \cdot \omega)=(2 \pi)^{\frac{n}{2}} \frac{J_{\frac{n}{n}}(r)}{r^{\frac{n}{2}}}$ and so we have $\mathcal{F} \chi_{\Omega_{0}}(\rho \cdot \omega)=0$ for any $\omega \in S^{n-1}$, that is, $\mathcal{N}\left(\Omega_{0}\right) \supset S(0: \rho)$.

Conversely, if $t \geq 0$ satisfies $\mathcal{N}\left(\Omega_{t}\right) \supset S(0: \rho)$. Then we have $\mathcal{F} \chi_{\Omega_{t}}(\rho \cdot \omega)=$ $\mathcal{F} \chi_{\Omega_{0}}(\rho \cdot \omega)=0$ for $\omega \in S^{n-1}$. Using the inequality (3.4.4), we have

$$
t\left|\frac{\partial}{\partial t}\right|_{t=0} \mathcal{F} \chi_{\Omega_{t}}(\rho \cdot \omega) \mid \leq t^{1+\alpha} \omega_{n-1} Y(g: \rho, T, \alpha) .
$$

Taking the $L^{2}$-norm on $S^{n-1}$, we have from (2.6.2) the following inequality:

$$
t \frac{(2 \pi)^{\frac{n}{2}}}{\rho^{\frac{n}{2}-1}}\left|g_{t}(0, \cdot)\right|_{\rho}^{\prime} \leq t^{1+\alpha} \omega_{n-1}{ }^{\frac{3}{2}} Y(g: \rho, T, \alpha) .
$$

This implies $t=0$ or $\frac{(2 \pi)^{\frac{n}{2}}\left|g_{t}(0, \cdot)\right|_{\rho}^{\prime}}{\rho^{\frac{n}{2}-1} \omega_{n-1} \frac{3}{2} Y(g: \rho, T, \alpha)} \leq t^{\alpha}$. Thus we have completed the proof. 
Lemma 4.2. Let $0<T, 0<\alpha \leq 1$ and $g:[0, T] \times S^{n-1} \rightarrow \mathbb{R}_{+}$be a $C^{1, \alpha}$ map such that $g(0, \eta) \equiv 1$ for $\eta \in S^{n-1}$. We put

$$
B \equiv B(g):=\|g\|_{\infty}^{n-1}\|g\|_{0+1} \omega_{n-1} .
$$

We recall notations of Lemma 2.3 such as $\varepsilon(n, R), \delta(n, R), k_{R}$ and $j\left(\frac{n}{2}, k\right)$. Let $R>j\left(\frac{n}{2}, 1\right)$. Assume

$$
\begin{aligned}
0 & \leq r_{0} \leq R, \\
0 & \leq t_{0}<\min \left(\frac{\varepsilon(n, R) \delta(n, R)}{B(g)}, T\right), \\
\omega_{0} & \in S^{n-1}, \\
\mathcal{F} \chi_{\Omega_{t_{0}}}\left(r_{0} \cdot \omega_{0}\right)=0 . &
\end{aligned}
$$

Then there exist $k \in\left\{1,2, \ldots, k_{R}\right\}$ and $a \in \mathbb{R}$ such that

$$
\begin{aligned}
r_{0} & =\left(1+a t_{0}\right) j\left(\frac{n}{2}, k\right), \\
|a| & \leq \frac{B(g)}{\varepsilon(n, R)}, \\
\left|r_{0}-j\left(\frac{n}{2}, k\right)\right| & <\delta(n, R) .
\end{aligned}
$$

Proof. If $t_{0}=0$ then we can take $a=0$. Hence we assume $t_{0}>0$. It follows from Lemma 3.4 that

$$
\left|\mathcal{F} \chi_{\Omega_{t_{0}}}\left(r_{0} \cdot \omega_{0}\right)-\mathcal{F} \chi_{\Omega_{0}}\left(r_{0} \cdot \omega_{0}\right)\right| \leq B(g) t_{0} .
$$

Since $\mathcal{F} \chi_{\Omega_{t_{0}}}\left(r_{0} \cdot \omega_{0}\right)=0$ and $\mathcal{F} \chi_{\Omega_{0}}\left(r_{0} \cdot \omega_{0}\right)=f\left(r_{0}\right)$ (see (2.3.1) for the definition of $f$ ), we have

$$
\left|f\left(r_{0}\right)\right| \leq B(g) t_{0}<\varepsilon(n, R) \delta(n, R) .
$$

Therefore, from Lemma 2.3 , we find $k \in\left\{1, \ldots, k_{R}\right\}$ such that

$$
\varepsilon(n, R)\left|r_{0}-j\left(\frac{n}{2}, k\right)\right| \leq\left|f\left(r_{0}\right)\right| \text {. }
$$

Now, we put $a:=\frac{r_{0}-j\left(\frac{n}{2}, k\right)}{t_{0} j\left(\frac{n}{2}, k\right)}$. Then we have

$$
|a| \leq \frac{\left|r_{0}-j\left(\frac{n}{2}, k\right)\right|}{t_{0}} \leq \frac{B(g)}{\varepsilon(n, R)}
$$


Here, the second inequality follows from (4.2.4) and (4.2.5), which proves (4.2.3b). The inequality (4.2.3c) is direct from (4.2.4) and (4.2.5).

Lemma 4.3. Retain the setting of Lemma 4.2. Assume

$$
0 \leq r_{0} \leq R, \quad 1 \leq R
$$

$$
0 \leq t_{0}<\min \left(\frac{\varepsilon(n, R) \delta(n, R)}{B(g)},\left(\frac{(2 \pi)^{\frac{n}{2}} \varepsilon(n, R)^{2}[g]_{R, \alpha}}{2^{n+4} n \omega_{n-1}{ }^{\frac{1}{2}} R^{\frac{n}{2}} B(g)^{3}}\right)^{\frac{1}{\alpha}}, T, 1\right),
$$

$$
\mathcal{N}\left(\Omega_{t_{0}}\right) \supset S\left(0: r_{0}\right) \text {. }
$$

Then $t_{0}=0$.

Proof. It follows from Lemma 4.2 that we find $a \in \mathbb{R}$ and $k \in\left\{1, \ldots, k_{R}\right\}$ such that

$$
\begin{aligned}
r_{0} & =\left(1+a t_{0}\right) j\left(\frac{n}{2}, k\right), \\
|a| & \leq \frac{B(g)}{\varepsilon(n, R)} .
\end{aligned}
$$

We put $\tilde{g}(t, \eta, a):=\left(1+a t_{0}\right) g(t, \eta)$. Then we have

$$
\mathcal{N}\left(\Omega\left(\tilde{g}\left(t_{0}, \cdot, a\right)\right)\right) \supset S\left(0: j\left(\frac{n}{2}, k\right)\right),
$$

because

$$
\begin{aligned}
\mathcal{F} \chi_{\Omega\left(\left(1+a t_{0}\right) g\left(t_{0}, \cdot\right)\right)}\left(j\left(\frac{n}{2}, k\right) \cdot \omega\right) & =\left(1+a t_{0}\right)^{n} \mathcal{F} \chi_{\Omega\left(g\left(t_{0}, \cdot\right)\right)}\left(\left(1+a t_{0}\right) j\left(\frac{n}{2}, k\right) \cdot \omega\right) \\
& =\left(1+a t_{0}\right)^{n} \mathcal{F} \chi_{\Omega\left(g\left(t_{0}, \cdot\right)\right)}\left(r_{0} \cdot \omega\right) .
\end{aligned}
$$

We want to apply Lemma 4.1 to $\tilde{g}(\cdot, \cdot, a)$ in order to show (4.3.3) implies $t_{0}=0$. To do this, it suffices to show (4.3.1b) implies (4.1.1) with $g$ replaced by $\tilde{g}$, that is,

$$
\frac{(2 \pi)^{\frac{n}{2}} \varepsilon(n, R)^{2}[g]_{R, \alpha}}{2^{n+4} n \omega_{n-1}{ }^{\frac{1}{2}} R^{\frac{n}{2}} B(g)^{3}} \leq \frac{(2 \pi)^{\frac{n}{2}}\left|\tilde{g}_{t}(0, \cdot, a)\right|_{\rho}^{\prime}}{\rho^{\frac{n}{2}-1} \omega_{n-1} \frac{3}{2} Y(\tilde{g}: \rho, T, \alpha)},
$$


where $\rho=j\left(\frac{n}{2}, k\right)(\leq R)$. Since $|a| \leq \frac{B(g)}{\varepsilon(n, R)}, t_{0} \leq 1$ and $t_{0} \leq \frac{\varepsilon(n, R) \delta(n, R)}{B(g)} \leq \frac{\varepsilon(n, R)}{B(g)}$ (see (2.4.1)), we can apply Lemma 3.5 and thus we have for $0<\rho \leq R$,

$$
\begin{aligned}
Y(\tilde{g}(\cdot, \cdot, a): \rho, T, \alpha) & \leq 2^{n+1}(n+R)\|g\|_{\infty}^{n-1}\|g\|_{0+\alpha}\|g\|_{1+\alpha}\left(1+\frac{B(g)}{\varepsilon(n, R)}\right)^{2} \\
& \leq 2^{n+2} n R \frac{B(g)}{\omega_{n-1}}\|g\|_{1+\alpha} 2^{2} \frac{B(g)^{2}}{\varepsilon(n, R)^{2}} \\
& =\frac{2^{n+4} n R B(g)^{3}\|g\|_{1+\alpha}}{\omega_{n-1} \varepsilon(n, R)^{2}}
\end{aligned}
$$

Here we have used $R \geq 1$ and $\varepsilon(n, R) \leq \omega_{n-1} \leq B(g)$ (see (2.4.2)). Therefore,

$$
\begin{aligned}
\frac{(2 \pi)^{\frac{n}{2}} \varepsilon(n, R)^{2}[g]_{R, \alpha}}{2^{n+4} n \omega_{n-1} 1^{\frac{1}{2}} R^{\frac{n}{2}} B(g)^{3}} & \leq \frac{(2 \pi)^{\frac{n}{2}}\left|g_{t}(0, \cdot)\right|_{R}}{R^{\frac{n}{2}-1} \omega_{n-1} \frac{3}{2} Y(\tilde{g}(\cdot, \cdot, a): \rho, T, \alpha)} \\
& =\frac{(2 \pi)^{\frac{n}{2}}\left|\tilde{g}_{t}(0, \cdot, a)\right|_{R}}{R^{\frac{n}{2}-1} \omega_{n-1} \frac{3}{2} Y(\tilde{g}(\cdot, \cdot, a): \rho, T, \alpha)} .
\end{aligned}
$$

Hence we have shown (4.3.4) and thus Lemma 4.3.

Proposition 4.4. Let $0<\alpha \leq 1,0<T$ and $0<R$. Suppose $g:[0, T] \times$ $S^{n-1} \longrightarrow \mathbb{R}_{+}$is a $C^{1, \alpha}$ map such that $g(0, \cdot) \equiv 1$. Retain the notation as in Lemma 4.3. Assume

$$
0<r, x \in \mathbb{R}^{n}
$$

$$
0 \leq t_{0}<\min \left(\frac{\varepsilon(n, R) \delta(n, R)}{B(g) e^{\|g\|_{\infty}}},\left(\frac{(2 \pi)^{\frac{n}{2}} \varepsilon(n, R)^{2}[g]_{R, \alpha}}{2^{n+4} n \omega_{n-1}{ }^{\frac{1}{2}} R^{\frac{n}{2}} B(g)^{3}}\right)^{\frac{1}{\alpha}}, T, 1\right)
$$

$$
\mathcal{N}\left(\Omega_{t_{0}}\right)_{\mathbb{R}} \cap B(0: R) \supset S(x: r) .
$$

Then $x=0$ and $t_{0}=0$.

Proof. It suffices to show $x=0$ by virtue of Lemma 4.3. Because $t_{0}$ satisfies (4.4.1b), the inequality (3.4.3) gives

$$
\left|\mathcal{F} \chi_{\Omega_{t_{0}}}(\zeta \cdot \omega)-\mathcal{F} \chi_{\Omega_{0}}(\zeta \cdot \omega)\right| \leq t_{0} B(g) e^{|\operatorname{Im} \zeta|\|g\|_{\infty}}<\varepsilon(n, R) \delta(n, R),
$$


for $\zeta \in \mathbb{C}$ with $|\operatorname{Im} \zeta| \leq 1$ and $\omega \in S^{n-1}$. On the other hand, if $\zeta \in \mathbb{C}$ satisfies $\left|\zeta-j\left(\frac{n}{2}, k\right)\right|=\delta(n, R)$ for some $k \in\left\{1, \ldots, k_{R}\right\}$, then

$$
\left|\mathcal{F} \chi_{\Omega_{0}}(\zeta \cdot \omega)\right| \geq \varepsilon(n, R) \delta(n, R)
$$

from Lemma 2.3. Therefore by using Rouche's theorem $\mathcal{F} \chi_{\Omega_{t_{0}}}(\zeta \cdot \omega)$ has a unique zero in $\left\{\zeta \in \mathbb{C}:\left|\zeta-j\left(\frac{n}{2}, k\right)\right|<\delta(n, R)\right\}$ for each $\omega \in S^{n-1}$.

Next, the condition (4.4.1c) means $\mathcal{F} \chi_{\Omega_{t_{0}}}(x+r \cdot \omega)=0$ for any $\omega \in S^{n-1}$. Applying Lemma 4.2 with $r_{0}=\|x+r \cdot \omega\|(\leq R)$ (with the notation there), we find $k \in\left\{1, \ldots, k_{R}\right\}$ for each $\omega \in S^{n-1}$ such that

$$
\left|\|x+r \cdot \omega\|-j\left(\frac{n}{2}, k\right)\right|<\delta(n, R) .
$$

By (2.3.2) and because $S^{n-1}$ is connected, the above integer $k$ does not depend on $\omega \in S^{n-1}$. This means that there exists $k \in\left\{1, \ldots, k_{R}\right\}$ such that

$$
\left|\|x+r \cdot \omega\|-j\left(\frac{n}{2}, k\right)\right|<\delta(n, R)
$$

for any $\omega \in S^{n-1}$.

Now, let us show $x=0$. Suppose $x \neq 0$. We put

$$
z_{+}:=\left(1+\frac{r}{\|x\|}\right) x, \quad z_{-}:=\left|1-\frac{r}{\|x\|}\right| x .
$$

Then, from (4.4.4) we have

$$
\left|\left\|z_{ \pm}\right\|-j\left(\frac{n}{2}, k\right)\right|<\delta(n, R) .
$$

On the other hand, since $\overline{\mathcal{F} \chi_{\Omega_{t_{0}}}(\zeta \cdot \omega)}=\mathcal{F} \chi_{\Omega_{t_{0}}}(-\zeta \cdot \omega)$ for $\zeta \in \mathbb{R}, \mathcal{N}\left(\Omega_{t_{0}}\right) \supset$ $S(x: r)$ if and only if $\mathcal{N}\left(\Omega_{t_{0}}\right) \supset S(-x: r)$. Therefore $\mathcal{F} \chi_{\Omega_{t_{0}}}( \pm x \pm r \cdot \omega)=0$ for any $\omega \in S^{n-1}$. In particular, we have

$$
\mathcal{F} \chi_{\Omega_{t_{0}}}\left(z_{ \pm}\right)=0
$$

Because $\mathcal{F} \chi_{\Omega_{t_{0}}}\left(\zeta \cdot \frac{x}{\|x\|}\right)$ has a unique zero in $\left\{\zeta \in \mathbb{C}:\left|\zeta-j\left(\frac{n}{2}, k\right)\right|<\delta(n, R)\right\}$, we conclude $\left\|z_{+}\right\|=\left\|z_{-}\right\|$. This implies $r=0$, which contradicts to (4.4.1a). Therefore $x$ must be the origin. Now Lemma 4.4 is reduced to Lemma 4.3.

In the normalized case where $\left\|g_{t}(0, \cdot)\right\|_{L^{\infty}\left(S^{n-1}\right)} \leq 1$, we can replace the assumption (4.4.1b) by a simpler one: 
Lemma 4.5. We put

$$
C_{n}:=\frac{1}{\omega_{n-1} \frac{1}{2}} \min \left(1, \frac{1}{2^{n+3} \omega_{n-1}}, \frac{(2 \pi)^{\frac{n}{2}}}{2^{4 n+7} n \omega_{n-1}^{3}}\right)
$$

For $(1<) j\left(\frac{n}{2}, 1\right) \leq R$, we put

$$
C(n, R):=\frac{C_{n} \varepsilon(n, R)^{2}}{R^{\frac{n}{2}}}
$$

where $\varepsilon(n, R)$ is the constant in Lemma (2.3). Let $0<T$ and $0<\alpha \leq 1$.

Suppose $g:[0, T] \times S^{n-1} \longrightarrow \mathbb{R}_{+}$is a $C^{1, \alpha}$ map such that

$$
\begin{gathered}
g(0, \eta) \equiv 1, \quad \text { for any } \eta \in S^{n-1}, \\
\sup _{\eta \in S^{n-1}}\left|g_{t}(0, \eta)\right| \leqq 1 .
\end{gathered}
$$

If $t$ satisfies

$$
0 \leq t<\min \left(T,\left(C(n, R)[g]_{R, \alpha}\right)^{\frac{1}{\alpha}}\right)
$$

then $t$ satisfies (4.4.1b).

Proof. If $r \geq 1$, then it follows from (2.6.3) that

$$
\left|g_{t}(0, \cdot)\right|_{r}^{\prime} \leq\left\|g_{t}(0, \cdot)\right\|_{L^{2}\left(S^{n-1}\right)} r^{\frac{1}{2}} \leq\left\|g_{t}(0, \cdot)\right\|_{L^{\infty}\left(S^{n-1}\right)} \omega_{n-1}{ }^{\frac{1}{2}} r^{\frac{1}{2}}
$$

Therefore, from the definition (2.6.4), we have

$$
[g]_{R, \alpha}=\frac{\left|g_{t}(0, \cdot)\right|_{R}}{\|g\|_{1+\alpha}} \leq \frac{\omega_{n-1}{ }^{\frac{1}{2}} R^{\frac{1}{2}}}{\|g\|_{1+\alpha}}
$$

Suppose $t$ satisfies (4.5.4). Then

$$
t^{\alpha}<\frac{C_{n} \varepsilon(n, R)^{2}}{R^{\frac{n}{2}}}[g]_{R, \alpha} \leq \frac{\omega_{n-1}{ }^{-\frac{1}{2}} \cdot 1}{R^{\frac{n}{2}}} \frac{\omega_{n-1}{ }^{\frac{1}{2}} R^{\frac{1}{2}}}{\|g\|_{1+\alpha}} \leq \frac{1}{\|g\|_{1+\alpha}} \leq \frac{1}{2} .
$$

Therefore we have

$$
\begin{aligned}
& t \leq \frac{1}{2} \\
& \left|g_{t}(t, \eta)\right| \leq\left|g_{t}(0, \eta)\right|+t^{\alpha}\|g\|_{1+\alpha} \leq 1+\frac{1}{2} \cdot 2=2 \text {, } \\
& |g(t, \eta)| \leq|g(0, \eta)|+t\left\|g_{t}\right\|_{\infty} \quad \leq 1+\frac{1}{2} \cdot 2=2 \\
& B(g)=\|g\|_{\infty}^{n-1}\|g\|_{0+1} \omega_{n-1} \leq 2^{n+1} \omega_{n-1}, \\
& B(g) e^{\|g\|_{\infty}} \leq 2^{n+1} \omega_{n-1} \cdot e^{2} \quad<2^{n+4} \omega_{n-1} \text {. }
\end{aligned}
$$


Thus from (4.5.2), (4.5.5) and (2.4.1) we have

$$
\begin{aligned}
t \leq t^{\alpha} & <C_{n} \frac{\varepsilon(n, R)^{2}}{R^{\frac{n}{2}}}[g]_{R, \alpha} \\
& \leq \frac{1}{2^{n+3} \omega_{n-1} \frac{3}{2}} \frac{\varepsilon(n, R) \delta(n, R)}{R^{\frac{n}{2}}} \frac{\omega_{n-1}{ }^{\frac{1}{2}} R^{\frac{1}{2}}}{\|g\|_{1+\alpha}} \\
& \leq \frac{\varepsilon(n, R) \delta(n, R)}{2^{n+4} \omega_{n-1}} \\
& \leq \frac{\varepsilon(n, R) \delta(n, R)}{B(g) e^{\|g\|_{\infty}}}
\end{aligned}
$$

On the other hand, since $C_{n} \leq \frac{(2 \pi)^{\frac{n}{2}}}{2^{4 n+7} n \omega_{n-1} \frac{7}{2}} \leq \frac{(2 \pi)^{\frac{n}{2}}}{B(g)^{3} 2^{n+4} n \omega_{n-1} \frac{1}{2}}$, we have

$$
\begin{aligned}
t^{\alpha} & <C_{n} \frac{\varepsilon(n, R)^{2}[g]_{R, \alpha}}{R^{\frac{n}{2}}} \\
& \leq \frac{(2 \pi)^{\frac{n}{2}} \varepsilon(n, R)^{2}[g]_{R, \alpha}}{2^{n+4} n \omega_{n-1}{ }^{\frac{1}{2}} B(g)^{3} R^{\frac{n}{2}}}
\end{aligned}
$$

Hence $t$ satisfies (4.4.1b).

Now, Theorem follows from Proposition 4.4 and Lemma 4.5. Corollary 1 is an immediate consequence of Theorem (with $x=0$ ) and Fact 1.1. Corollary 2 is a direct consequence of Corollary 1.

\section{Appendix (GEOMETRIC ASPECT OF UNESSENTIAL PERTURBATION)}

In Definition (1.5), we have defined a star-shaped region (with respect to the origin) by using the polar coordinate, that is, $\Omega(g) \subset \mathbb{R}^{n}$ for $g \in C\left(S^{n-1}, \mathbb{R}_{+}\right)$. In this section, we also consider a star-shaped region $\Omega$ by defining the boundary $G: S^{n-1} \rightarrow \partial \Omega$, and discuss a geometric aspect of "unessential perturbation" that we have treated. This is done in Proposition 5.7. Throughout this section we use the notation $C^{k}$ where $k$ stands for $l(l \in \mathbb{N}=\{0,1, \ldots\}),(l, \alpha)$ $(l \in \mathbb{N} ; 0<\alpha \leq 1), \infty$ or $\omega$ (real analytic). The condition $k \geq 1$ means that we exclude the cases $k=0$ and $k=(0, \alpha)$ from the above possibilities. We define a standard norm in $\mathbb{R}^{n}$ by $\|x\|:=\left(\sum_{j=1}^{n} x_{j}{ }^{2}\right)^{\frac{1}{2}}$ for $x={ }^{t}\left(x_{1}, \ldots, x_{n}\right) \in \mathbb{R}^{n}$. 
Definition 5.1. A $C^{k}$-map $G: S^{n-1} \rightarrow \mathbb{R}^{n}$ is called to bound a $C^{k}$ star-shaped region (with respect to the origin) if the following two conditions are satisfied:

$$
\text { Image }(G) \not \supset 0
$$

$$
\frac{G}{\|G\|}: S^{n-1} \rightarrow S^{n-1}, \omega \mapsto \frac{G(\omega)}{\|G(\omega)\|} \text { defines a } C^{k} \text {-diffeomorphism of } S^{n-1} \text {. }
$$

Then Image $(G)$ is the boundary of a star-shaped region (see Lemma $5.2(1)$ ). We write $C_{\star}^{k}\left(S^{n-1}, \mathbb{R}^{n}\right)$ for the totality of such maps.

Clearly, if $h \in C^{k}$-Diffeo $\left(S^{n-1}\right)$ and $G \in C_{\star}^{k}\left(S^{n-1}, \mathbb{R}^{n}\right)$, then both $G$ and $G \circ h$ define the same boundary $\operatorname{Image}(G)=\operatorname{Image}(G \circ h)$. The following lemma relates Definition (1.5) with Definition 5.1.

Lemma 5.2. We have a bijection:

$$
C_{\star}^{k}\left(S^{n-1}, \mathbb{R}^{n}\right) \simeq C^{k}\left(S^{n-1}, \mathbb{R}_{+}\right) \times C^{k}-\operatorname{Diffeo}\left(S^{n-1}\right), \quad G \mapsto\left(g_{G}, h_{G}\right),
$$

characterized by the following property:

$$
G \circ h_{G}(\eta)=g_{G}(\eta) \eta \quad\left(\eta \in S^{n-1}\right) .
$$

In particular,

1) Image $(G)=\partial \Omega\left(g_{G}\right)$, the boundary of the star-shaped region $\Omega\left(g_{G}\right)$ (see (1.5)).

2) If $G_{1}, G_{2} \in C_{\star}^{k}\left(S^{n-1}, \mathbb{R}^{n}\right)$ define the same boundary Image $\left(G_{1}\right)=$ Image $\left(G_{2}\right)$, then there exists an $h \in C^{k}-\operatorname{Diffeo}\left(S^{n-1}\right)$ such that $G_{1} \circ h=$ $G_{2}$.

Proof. Given $G \in C_{\star}^{k}\left(S^{n-1}, \mathbb{R}^{n}\right)$, we put $h_{G}:=\left(\frac{G}{\|G\|}\right)^{-1} \in C^{k}$-Diffeo $\left(S^{n-1}\right)$ and $g_{G}(\eta):=\left\|G \circ h_{G}(\eta)\right\|\left(\eta \in S^{n-1}\right)$. It follows from definition of $h_{G}$ that $\frac{G\left(h_{G}(\eta)\right)}{\left\|G\left(h_{G}(\eta)\right)\right\|}=\eta$ and so $G \circ h_{G}(\eta)=g_{G}(\eta) \eta$. Conversely, given $(g, h) \in$ $C^{k}\left(S^{n-1}, \mathbb{R}_{+}\right) \times C^{k}$-Diffeo $\left(S^{n-1}\right)$, we define $G(\omega):=g\left(h^{-1}(\omega)\right) h^{-1}(\omega)$. Then it follows from definition that $G \in C_{\star}^{k}\left(S^{n-1}, \mathbb{R}^{n}\right)$ and that $\left(g_{G}, h_{G}\right)=(g, h)$. This establishes the bijection (5.2.1). The remaining part (1) is followed from (5.2.2), and (2) holds if we put $h_{G}:=h_{G_{1}} \circ h_{G_{2}}^{-1}$. 
Next, we consider a deformation of domains by $\operatorname{Map}\left([0, T], C_{\star}^{k}\left(S^{n-1}, \mathbb{R}^{n}\right)\right)$. We prepare the following lemma asserting that it is an open condition for a domain to be star-shaped under a $C^{1}$-deformation of domains.

Lemma 5.3. Let $k \geq 1$. Suppose $G:[0, T] \times S^{n-1} \rightarrow \mathbb{R}^{n}$ is a $C^{k}$-map such that $G(0, \cdot) \in C_{\star}^{k}\left(S^{n-1}, \mathbb{R}^{n}\right)$ (see Definition 5.1 ). Then there exists a positive number $0<T^{\prime} \leq T$ such that $G(t, \cdot) \in C_{\star}^{k}\left(S^{n-1}, \mathbb{R}^{n}\right)$ for any $t \in\left[0, T^{\prime}\right]$. Moreover, if we define $g_{G}(t, \eta)=g_{G(t, \cdot)}(\eta)$ and $h_{G}(t, \eta)=h_{G(t, \cdot)}(\eta)$ with the notation in Lemma 5.2, then $g_{G}:\left[0, T^{\prime}\right] \times S^{n-1} \rightarrow \mathbb{R}_{+}$and $h_{G}:\left[0, T^{\prime}\right] \times S^{n-1} \rightarrow$ $S^{n-1}$ are $C^{k}$-maps satisfying

(5.3.1) $h_{G}(t, \cdot)$ is a $C^{k}$-diffeomorphism of $S^{n-1}$ for $t \in\left[0, T^{\prime}\right]$.

(5.3.2) $G\left(t, h_{G}(t, \eta)\right)=g_{G}(t, \eta) \eta$ for $t \in\left[0, T^{\prime}\right], \eta \in S^{n-1}$.

Proof. We fix $T^{\prime \prime}>0$ such that $G\left(\left[0, T^{\prime \prime}\right] \times S^{n-1}\right) \subset \mathbb{R}^{n} \backslash\{0\}$. We define a $C^{k}$. $\operatorname{map} H:\left[0, T^{\prime \prime}\right] \times S^{n-1} \rightarrow S^{n-1}$ by $H(t, \omega):=\frac{G(t, \omega)}{\|G(t, \omega)\|}$ for $(t, \omega) \in\left[0, T^{\prime \prime}\right] \times S^{n-1}$. Let us show that the map $H(t, \cdot): S^{n-1} \rightarrow S^{n-1}$ is a $C^{k}$-diffeomorphism for any small $t$.

Since $G(0, \cdot)$ bounds a star-shaped region, we find $C^{k}$-maps $h: S^{n-1} \rightarrow S^{n-1}$ and $g: S^{n-1} \rightarrow \mathbb{R}_{+}$such that $G(0, h(\eta))=g(\eta) \eta$ by Lemma 5.2. Then the Jacobian $J H(0, \cdot)$ of the map $H(0, \cdot)=h^{-1}: S^{n-1} \rightarrow S^{n-1}$ is nowhere vanishing. Because $H(t, \omega)$ is in the $C^{k}$ class and because $S^{n-1}$ is compact, there exists $\left(T^{\prime \prime} \geq\right) T^{\prime}>0$ such that the Jacobian $J H(t, \cdot)$ of the map $H(t, \cdot): S^{n-1} \rightarrow S^{n-1}$ is nowhere vanishing for all $t \in\left[0, T^{\prime}\right]$. This means that the map $H(t, \cdot): S^{n-1} \rightarrow S^{n-1}$ is locally $C^{k}$-diffeomorphic. Because a local homeomorphism from a compact manifold to a connected one is automatically a covering map, the map $H(t, \cdot): S^{n-1} \rightarrow S^{n-1}$ is a covering map. On the other hand, consider the following commutative diagram of homology groups:

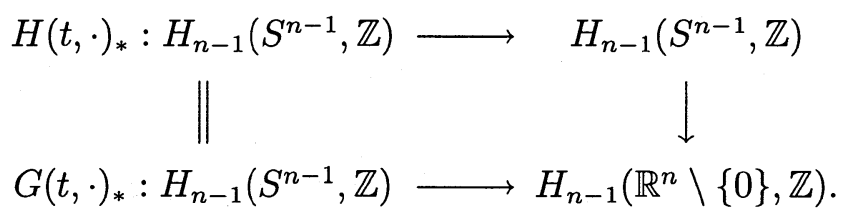

Since $G(t, w)$ is continuous, the mapping degree of $H(t, \cdot)$ equals to that of $H(0, \cdot)$, that is, \pm 1 . Therefore $H(t, \cdot)$ is a $C^{k}$-diffeomorphism from $S^{n-1}$ to $S^{n-1}$ for any $t \in\left[0, T^{\prime}\right]$. Hence $G(t, \cdot) \in C_{\star}^{k}\left(S^{n-1}, \mathbb{R}^{n}\right)$ for any $t \in\left[0, T^{\prime}\right]$ 
by definition. Now the last statement is proved in the same way as Lemma 5.2 .

A deformation of domains starting from a unit ball is a special case of the previous lemma and so we have:

Lemma 5.4. Let $k \geq 1$. Suppose $G:[0, T] \times S^{n-1} \rightarrow \mathbb{R}^{n}$ is a $C^{k}$-map such that $G(0, \omega) \equiv \omega\left(\omega \in S^{n-1}\right)$. Then there exist a positive number $0<T^{\prime} \leq T$, and $C^{k}-$ maps $g_{G}:\left[0, T^{\prime}\right] \times S^{n-1} \rightarrow \mathbb{R}_{+}$and $h_{G}:\left[0, T^{\prime}\right] \times S^{n-1} \rightarrow S^{n-1}$ satisfying (5.3.1), (5.3.2) and

$$
g_{G}(0, \eta) \equiv 1, h_{G}(0, \eta) \equiv \eta \quad \text { for } \eta \in S^{n-1}
$$

Thus the map $G$ defines a family of star-shaped regions

$$
\Omega_{t}:=\Omega\left(g_{G}(t, \cdot)\right) \quad(\text { see }(1.5))
$$

parametrized by $t \in\left[0, T^{\prime}\right]$ with $\Omega_{0}=B(0: 1)$, the unit ball.

Let $T \mathbb{R}^{n} \rightarrow \mathbb{R}^{n}$ be the tangent bundle, and $T \mathbb{R}^{n}{ }_{\mid S^{n-1}} \rightarrow S^{n-1}$ its restriction to $S^{n-1}$. We write $\Gamma^{k}\left(T \mathbb{R}^{n}{ }_{\mid S^{n-1}}\right)$ for the space of sections in the $C^{k}$ class.

Definition 5.2. Suppose $G:[0, T] \times S^{n-1} \rightarrow \mathbb{R}^{n}$ is a $C^{1}$-map such that $G(0, \omega) \equiv \omega\left(\omega \in S^{n-1}\right)$. We define the velocity $X_{G} \in \Gamma^{1}\left(\left.T \mathbb{R}^{n}\right|_{S^{n-1}}\right)$ of a deformation $\Omega_{t}=\Omega\left(g_{G}(t, \cdot)\right)$ (see (5.4.2)) by

$$
X_{G}(\omega):=\left.\frac{\partial}{\partial t}\right|_{t=0} G(t, \omega) .
$$

The map $G(t, \omega)$ is called degenerate at $t=0$ if $X_{G}(\omega) \equiv 0\left(\omega \in S^{n-1}\right)$.

The groups $C^{k}$-Diffeo $\left(S^{n-1}\right)$ and $A F\left(\mathbb{R}^{n}\right):=G L(n, \mathbb{R}) \ltimes \mathbb{R}^{n}$ act naturally on $C^{k}\left(S^{n-1}, \mathbb{R}^{n}\right)$ by $G \mapsto \pi \circ G \circ \varphi$, where $\varphi \in C^{k}$-Diffeo $\left(S^{n-1}\right), \pi \in A F\left(\mathbb{R}^{n}\right)$ and $G \in C^{k}\left(S^{n-1}, \mathbb{R}^{n}\right)$. In particular, if we define $\pi(a, b) \in A F\left(\mathbb{R}^{n}\right)(a>$ $\left.0, b \in \mathbb{R}^{n}\right)$ by $\pi(a, b) x:=a x+b\left(x \in \mathbb{R}^{n}\right)$ describing a parallel translation and similarity transform, then $(\pi(a, b) \circ G \circ \varphi)(\omega)=a G(\varphi(\omega))+b$.

As we are interested in the perturbation of domains up to parallel translations and similarity transforms, we consider: 
Definition 5.3. Suppose $G_{i}:\left[0, T_{i}\right] \times S^{n-1} \rightarrow \mathbb{R}^{n}$ is a $C^{k}$-map such that $G(0, \omega) \equiv \omega(i=1,2)$. We call $G_{1}$ is $C^{k}$-equivalent to $G_{2}$ if there exist $0<T^{\prime \prime} \leq T$ and $C^{k}$-maps

$$
\begin{cases}a:\left[0, T^{\prime \prime}\right] \rightarrow \mathbb{R}, & a(0)=1 \\ b:\left[0, T^{\prime \prime}\right] \rightarrow \mathbb{R}^{n}, & b(0)={ }^{t}(0, \ldots, 0) \\ \varphi:\left[0, T^{\prime \prime}\right] \times S^{n-1} \rightarrow S^{n-1}, & \varphi(0, \omega) \equiv \omega \\ & \varphi(t, \cdot) \text { is a } C^{k} \text {-diffeomorphism of } \\ S^{n-1}\end{cases}
$$

such that $G_{2}(t, \cdot)=\pi(a(t), b(t)) \circ G_{1}(t, \cdot) \circ \varphi(t, \cdot)$, that is,

$$
G_{2}(t, \omega)=a(t) G_{1}(t, \varphi(t, \omega))+b(t)
$$

for $t \in\left[0, T^{\prime}\right], \omega \in S^{n-1}$. We write $[G]^{k}$ for the $C^{k}$-equivalence class containing $G$.

Proposition 5.7. Let $k \geq 1$. Suppose $G:[0, T] \times S^{n-1} \rightarrow \mathbb{R}^{n}$ is a $C^{k}$-map such that $G(0, \omega) \equiv \omega$. Let $g_{G}:\left[0, T^{\prime}\right] \times S^{n-1} \rightarrow \mathbb{R}_{+}$be the corresponding function in Lemma 5.3. Then the following conditions on the map $G$ are equivalent:

(1) $\Omega\left(g_{G}(t, \cdot)\right)$ is an unessential family of perturbations (see $\S 1$ ).

(2) Among the $C^{k}$-equivalence class $[G]^{k}$ (Definition 5.6), there exists $\widetilde{G}$ which is degenerate at $t=0$ (see Definition 5.5).

The rest of this section is devoted to the proof of Proposition 5.7.

Let $T_{S^{n-1}} \mathbb{R}^{n} \rightarrow S^{n-1}$ be the normal bundle associated to the embedding of Riemannian manifolds $S^{n-1} \subset \mathbb{R}^{n}$. According to a Whitney sum of vector bundles over $S^{n-1}$

$$
T \mathbb{R}^{n}{ }_{\mid S^{n-1}}=T S^{n-1} \oplus T_{S^{n-1}} \mathbb{R}^{n}
$$

we have a direct sum of vector spaces:

$$
\Gamma^{k}\left(T \mathbb{R}^{n}{ }_{\mid S^{n-1}}\right)=X^{k}\left(S^{n-1}\right) \oplus C^{k}\left(S^{n-1}\right),
$$

where we denote by $X^{k}\left(S^{n-1}\right)$ the space of vector fields over $S^{n-1}$ in the $C^{k}$ class, and identify $C^{k}\left(S^{n-1}\right)$ with $\Gamma^{k}\left(T_{S^{n-1}} \mathbb{R}^{n}\right)$ by the outer normal vector field. We recall $E_{0}, E_{1}$ are the subspace of $C^{\omega}\left(S^{n-1}\right)$ which are the eigenspaces of the Laplace-Beltrami operator $\Delta_{S^{n-1}}$ with eigenvalues $0,1-n$, respectively 
(see Lemma 2.5). Thus we regard $X^{k}\left(S^{n-1}\right) \oplus E_{0} \oplus E_{1} \subset \Gamma^{k}\left(T \mathbb{R}^{n}{ }_{\mid S^{n-1}}\right)$. We write a quotient map as

$$
\begin{aligned}
p: \Gamma^{k}\left(T \mathbb{R}_{\mid S^{n-1}}^{n}\right) \rightarrow & \Gamma^{k}\left(T \mathbb{R}^{n}{ }_{\mid S^{n-1}}\right) /\left(X^{k}\left(S^{n-1}\right) \oplus E_{0} \oplus E_{1}\right) \\
& \simeq C^{k}\left(S^{n-1}\right) \cap\left(\sum_{k=2}^{\infty} \oplus E_{k}\right) .
\end{aligned}
$$

Formula 5.9. Suppose $T>0$ and we are given $C^{1}$-maps

$$
\begin{array}{ll}
G:[0, T] \times S^{n-1} \rightarrow \mathbb{R}^{n}, & G(0, \omega) \equiv \omega\left(\omega \in S^{n-1}\right), \\
a:[0, T] \rightarrow \mathbb{R}, & a(0)=1, \\
b:[0, T] \rightarrow \mathbb{R}^{n}, & b(0)={ }^{t}(0, \ldots, 0), \\
\varphi:[0, T] \times S^{n-1} \rightarrow S^{n-1}, & \varphi(0, \omega) \equiv \omega .
\end{array}
$$

If we put $\widetilde{G}(t, \omega):=a(t) G(t, \varphi(t, \omega))+b(t)$, then with the notation in Definition 5.5

$$
X_{\widetilde{G}}(\omega)=X_{G}(\omega)+X_{\varphi}(\omega)+a^{\prime}(0) \omega+b^{\prime}(0)
$$

In particular, $p\left(X_{G}\right) \in C^{k}\left(S^{n-1}\right) \cap \sum_{k=2}^{\infty} \oplus E_{k}$ is determined only by the equivalent class $[G]^{k}$.

Proof. The formula (5.9.1) follows by a direct calculation.

Proof of Proposition 5.7. In the setting of Proposition 5.7, we consider another condition on the map $G$ :

$$
p\left(X_{G}\right)=0 \text {, that is, } X_{G} \in X^{k}\left(S^{n-1}\right) \oplus E_{0} \oplus E_{1} .
$$

i) $(5.7 .1) \Leftrightarrow(5.7 .3)$ :

Because $G\left(t, h_{G}(t, \eta)\right)=g_{G}(t, \eta) \eta$, we have

$$
\left.\frac{\partial}{\partial t}\right|_{t=0} G\left(t, h_{G}(t, \eta)\right)=\left.\frac{\partial}{\partial t}\right|_{t=0} g_{G}(t, \eta) \eta,
$$

that is, $X_{G}(\eta)+X_{h_{G}}(\eta)=g_{G}(\eta) \eta$ since $G(0, \eta)=h_{G}(0, \eta)=\eta$. Because $X_{h_{G}} \in X^{k}\left(S^{n-1}\right)$ and because $\left(\eta \mapsto g_{G}(\eta) \eta\right) \in \Gamma^{k}\left(T_{S^{n-1}} \mathbb{R}^{n}\right)$ is identified with $g_{G} \in C^{k}\left(S^{n-1}\right)$ via the outer normal vector field, we have

$$
p\left(X_{G}\right)=g_{G} \bmod E_{0} \oplus E_{1} .
$$


Therefore $p\left(X_{G}\right)=0$ if and only if $g_{G} \in E_{0} \oplus E_{1}$, that is, (5.7.3) is equivalent to (5.7.1).

ii) $(5.7 .2) \Leftrightarrow(5.7 .3)$ :

Assume there exists $\widetilde{G} \in[G]^{k}$ such that $X_{\widetilde{G}}=0$. It follows from Formula (5.9) that $p\left(X_{G}\right)=p\left(X_{\widetilde{G}}\right)=0$. Therefore, (5.7.2) implies (5.7.3). Conversely, assume $p\left(X_{G}\right)=0$, that is, $X_{G} \in X^{k}\left(S^{n-1}\right) \oplus E_{0} \oplus E_{1}$. In view of (2.5.1) and (2.5.2) we find $Y \in X^{k}\left(S^{n-1}\right)$ and $a \in \mathbb{R}, b \in \mathbb{R}^{n}$ such that

$$
X_{G}(\omega)=Y(\omega)+(a+(b, \omega)) \omega \quad\left(\omega \in S^{n-1}\right) .
$$

Because $S^{n-1}$ is compact, there exist $0<T^{\prime \prime} \leq T^{\prime}$ and a one parameter family of diffeomorphisms $\varphi:\left[0, T^{\prime \prime}\right] \times S^{n-1} \rightarrow S^{n-1}$ such that

$$
\left.\frac{\partial}{\partial t}\right|_{t=0} \varphi(t, \omega)=-Y(\omega)+(b-(b, \omega) \omega),
$$

from fundamental existence and uniqueness theorems of a system of first order differential equations. We put $a(t):=1-a t, b(t):=-b t$ and $\widetilde{G}(t, \omega):=$ $a(t) G(t, \varphi(t, \omega))+b(t)$. Then, from Formula (5.9.1), we have

$$
X_{\widetilde{G}}(\omega)=X_{G}(\omega)+(-Y(\omega)+b-(b, \omega) \omega)-a \omega-b=0 .
$$

Hence we have proved (5.7.3) implies (5.7.2)

Although the assumption in Corollary 2 about "not unessential" restricts the perturbation to "generic" cases, we can also treat "degenerate cases" (Definition 5.5, Proposition 5.7) by a change of variables if the degree of degeneracy is finite in the sense of (5.10.1). To be more precise, we mention:

Remark 5.10. Let $k=\infty$ or $\omega$ (real analytic). Given a $C^{k}$-map $G:[0, T] \times$ $S^{n-1} \rightarrow \mathbb{R}^{n}$ such that $G(0, \eta) \equiv \eta$. If $\Omega\left(g_{G}(t, \cdot)\right)$ is not unessential, we can apply Corollary 2. Otherwise, from the equivalent condition (5.7.2) we can find $\widetilde{G} \in[G]^{k}$ such that $\widetilde{G}(t, \eta)-\eta \equiv 0 \bmod t$, so that $G_{1}(t, \eta):=\widetilde{G}(\sqrt{t}, \eta)$ is in the $C^{1, \frac{1}{2}}$ class. In the same way, either we can apply Corollary 2 to $G_{1}$ or we can find $\widetilde{\widetilde{G}} \in[G]^{k}$ such that $\widetilde{\widetilde{G}}(t, \eta)-\eta \equiv 0 \bmod t^{2}$, so that $G_{2}(t, \eta):=\widetilde{\widetilde{G}}(\sqrt[3]{t}, \eta)$ is in the $C^{1, \frac{1}{3}}$ class. By an iteration of this argument, we can show either

(5.10.1) there exist $l \in \mathbb{N}$ and $\widetilde{G} \in[G]^{k}$ such that $\widetilde{G}(t, \eta)-\eta \equiv 0 \bmod t^{l}$ and that $\widetilde{G}\left(t^{\frac{1}{1+1}}, \eta\right) \in C^{1, \frac{1}{l+1}}\left(\left[0, T^{\prime}\right] \times S^{n-1}, \mathbb{R}^{n}\right)$ defines a family of domains which is not unessential, 
or

(5.10.2) for any $l \in \mathbb{N}$ there exists $G_{l} \in[G]^{k}$ such that $G_{l}(t, \eta)-\eta \equiv 0$ $\bmod t^{l}$.

In the real analytic case $k=\omega$, it is likely that (5.10.2) can be replaced by $(5.10 .2)^{\prime}$ there exists $\widetilde{G} \in[G]^{\omega}$ such that $\widetilde{G}(t, \eta) \equiv \eta$.

This would enable us to drop the assumption "not unessential" in Corollary 2 in the case where $G$ is real analytic, because the case $(5.10 .2)^{\prime}$ means that $\Omega\left(g_{G}(t, \cdot)\right)$ is a ball for all $t$ and because we can apply the original form of Corollary 2 in the case (5.10.1) to the map $\widetilde{G}$.

\section{ACKNOWLEDGEMENT}

The author would like to thank Professors C. Berenstein, B. Ørsted and T. Oshima for their comments and interest in this work. He is also grateful to Ms. Carol Warfield for her typing of the manuscript during his stay at the School of Mathematics of the Institute for Advanced Study at Princeton.

\section{REFERENCES}

1. Agranovsky, M. L., On the stability of the spectrum in the Pompeiu problem, Preprint.

2. Aviles, P., Symmetry theorems related to Pompeiu's problem, Amer. J. Math., 108-5 (1986), 1023-1036.

3. Berenstein, C. A., On the converse to Pompeiu problems, Notas e Communicacōes de Matemática (Univ. Fed. de Bernambuco), 73 (1976).

4. _ An inverse spectral theorem and its relation to the Pompeiu problem, J. Analyse Math., 37 (1980), 128-144.

5. __ An inverse Neumann problem, J. reine angew. Math., 382 (1987), 1-21.

6. Brown, L. and Kahane, J. P., A note on the Pompeiu problem for convex domains, Math. Ann., 259-1 (1982), 107- 110.

7. Brown, L., Schreiber, B. M. and Taylor, B. A., Spectral synthesis and the Pompeiu problem, Ann. Inst. Fourier (Grenoble), 23 ( 1973), 125-154.

8. Erdélyi, A., Magnus, W., Oberhettinger, F., and Tricomi, F. G., Higher Transcendental Functions, (Bateman Manuscript Projec), 2, McGraw-Hill, New York 1953.

9. Garofalo, N., and Segala, F., New results on the Pompeiu problem, Trans. Amer. Math. Soc., 325-1 (1991), 273-286.

10. Helgason, S., Groups and Geometric Analysis, Pure and Appl. Math., vol. 113 Academic Press, New York and London 1984. 
11. Kobayashi, T., Null varieties for convex domains, Reports on unitary representation seminar, 6 (1986), 1-18, Proceedings of the conference on "Unitary representation theory" held at Toba (in Japanese).

12. _ On characteristic functions of domains and the zeros of their FourierLaplace transforms, Master's dissertation II, University of Tokyo, 1987 (in Japanese).

13. __ Asymptotic behaviours of the null variety for a convex domain in a nonpositively curved space form, Jour. Fac. Sci. Univ. Tokyo, 36-3 (1989), 389-478.

14. __ Convex domains and Fourier transform on spaces of constant curvature, to appear in Lecture notes of the Centre International de Mathematiques Pures et Appliquees held at WuHan University in P.R. China in 1991 (112 pages).

15. Pompeiu, D., Sur certains systèms d'équations linéaires et sur une propriété intégrale des fonktions de plusieurs variables, C. R. Acad. Sci. Paris, 188 (1929), 1138-1139.

16. __ Sur une propriété intégrales des fonctions de deux variables réelles, Bull. Sci. Acad. Royale Belgique, 15 (1929), 265-269.

17. Watson, G. N., A Treatise on the Theory of Bessel Functions, Second edition Cambridge at the university press, 1962.

18. Williams, S. A., A partial solution of the Pompeiu problem, Math. Ann., 223-2 (1976), 183-190.

19. _ Analyticity of the boundary for Lipschitz domains without the Pompeiu property, Indiana Univ. Math. J., 30-3 (1981), 357-369.

20. Yau, S. T., Problem Section, in Seminar on Differential Geometry, edited S.T.Yau, Annals of Math. Studies, Princeton, N. J. 1982.

UNIVERSITY OF TOKYO, TOKYO, JAPAN.

E-mail address: toshi@tansei.cc.u-tokyo.ac.jp

ReCeived December 10, 1992 Revised May 6, 1993. 